\title{
Stimulus Pronunciability in Aural Paired-Associate Learning ${ }^{1}$
}

\author{
Edwin Martin \\ University of Michigan, Ann Arbor, Michigan
}

\begin{abstract}
An experiment was conducted in an attempt to assess the merits of a stimulus availability interpretation of stimulus pronunciability (PR) effects in aural paired-associate learning. Stimulus PR was varied within lists of 8 trigram-adjective pairs. The study-test technique was used. The intra-SR interval of study trials was either 2,4 , or $6 \mathrm{sec}$, thus allowing periods of different length in which to manipulate stimulus availability at the time of the response event. Subgroups of $S$ s either counted backwardly during this interval, repeated the stimulus aloud, or were left uninstructed. Stimulus recall was given at the end of learning without forewarning.

The results indicate that stimulus repetition efficiently serves to maintain availability over the intra-SR interval. Counting backwardly during this interval, however, markedly impedes acquisition, but not differentially from the stimulus-repetition condition over levels of PR as would be expected from short-term retention studies involving pronunciability as a variable. The failure of the three intra-SR activities to interact over levels of $P R$ both during acquisition and in post-acquisition stimulus recall makes differential availability (in the sense of recallability) according to $P R$ an untenable explanation of stimulus-PR effects.
\end{abstract}

It is evident from the literature that stimulus-term pronunciability (PR) is a significant variable in paired-associate acquisition: performance is directly related to ease of scaled PR (e.g., Underwood and Schulz, 1960; Martin and Schulz, 1963). This relationship obtains in spite of the fact that $S$ s are not required to recall, reproduce, pronounce, or in any way overtly utilize the variably pronounceable stimuli. The question therefore arises as to how it is that stimulus-term PR exerts its effect on performance.

One hypothesis is that the stimulus-PR effect is underlain by what is commonly termed availability. Such a view is attractive

1 This research was supported by the Advanced Research Projects Agency, Department of Defense, and was monitored by the Air Force Office of Scientific Research under Contract No. AF 49(638)1235 with the Human Performance Center, Department of Psychology. Data collection and parts of the analyses were carried out by Kelyn H. Roberts. for two reasons. First, it rounds out the twophase conception of verbal learning in a symmetric way: whereas ordinarily the total learning process is seen as analyzable into response learning (which results in response availability) and association formation, there is no a priori reason not to suppose that stimulus-learning should also be included. Indeed, unless the amply evident phenomenon of backward association formation is taken as different in kind from forward association formation or is denied as an integral part of the total paired-associate learning process, it would seem required in the interests of consistency to admit that stimulus learning is to backward association formation as response learning is to forward association formation, and that therefore stimulus learning must be included in a complete analysis of pairedassociate learning.

The second reason why the stimulus-availability hypothesis is attractive is that within 
the S-R framework, as least, it is patent that in order for $S$ to form an association between a stimulus and a response he must have the stimulus "in mind" when the response event occurs. This view of the learning process, together with the fact that retention of single verbal units varies directly with the meaningfulness of the units, seems to require the conclusion that the stimulus-PR effect is an availability phenomenon. Thus, in the aural paired-associate situation where stimulus and response events are clearly sequential, a difficult-to-pronounce stimulus should be less available to $S$ upon presentation of the response than an easy-to-pronounce stimulus. In other words, because difficult-to-pronounce units are less meaningful (Noble, 1963) and more rapidly forgotten over short intervals than are easy-to-pronounce units (Lindley, 1963; Murdock, 1961; Peterson, Peterson, and Miller, 1961), it follows that, unless the stimulus and response events are simultaneous, the opportunity exists for stimuli which are differentially pronounceable to be differentially lost prior to the response event. It might be added, parenthetically, that even with the approximate simultaneity of stimulus and response events in visual paired-associate learning, it is plausible to suppose that either event may contribute an effective differential availability to the assumed underlying association-formation process.

In an attempt to evaluate this hypothesis, an aural paired-associate experiment was conducted which provided for (a) within-lists variation of stimulus PR, (b) delays of different lengths between stimulus and response events, (c) different delay-period activities that either maximized or minimized the likelihood of stimulus forgetting, and (d) additional conditions in which the delay was either of zero length or of finite length but not filled with an instructed activity.

\section{Method}

Lists. Eight lists of eight trigram-adjective pairs each were constructed with ostensibly equivalent properties. This was done by drawing up four lists of trigrams and two lists of two-syllable adjectives and combining them in the eight possible ways. Each of the four lists of trigrams comprised two trigrams at each of the following mean PR levels (Underwood and Schulz, 1960): 1.93, 4.00, 6.32, and 8.52 . The adjectives were taken from Haagen (1942); all occur more often than 50 per million according to Thorndike and Lorge (1944). Intralist similarity was as minimal as seemed possible: within a list of trigrams, no letter was used more than twice (but never in the same position), and at that only when forced by the size of the pool of scaled trigrams; the adjectives were completely unrelated among themselves in the judgment of the investigator. In the pairing of the trigrams and adjectives, pairs of adjectives were counterbalanced over PR levels.

Design and Procedure. All $S$ s were given 15 study and 15 test trials in alternating order. The intra-SR interval (the time between presentations of stimulus and response members on study trials) was either 2,4 , or $6 \mathrm{sec}$ for various groups of $S$ s, thus allowing three periods of different lengths in which to manipulate stimulus availability.

The $S$ s assigned to each intra-SR interval were divided into three groups according to their studytrials intra-SR-interval activity. In Group $\mathrm{R}, S$ listened silently as $E$ spelled the stimulus; then, when an electric metronome was turned on at 3 beats/sec., $S$ began repeating aloud the trigram spelled by $E$, spelling in time with the metronome until it was turned off; after the metronome was turned off, $E$ said the response. For Group C, $E$ spelled the stimulus and then, upon the onset of the metronome, said a three-digit number; $S$ counted backwardly from that number by 3 s until the metronome was turned off, at which time $E$ said the response. The Group-C condition was thus optimal for stimulus loss (presumably differentially according to $P R$ ) prior to each response event, while the Group- $R$ condition provided for maintenance of stimulus availability.

The $S$ s of Group $U$ were treated identically with those of Groups $\mathbf{R}$ and $\mathbf{C}$ with respect to presentation of stimuli and responses and the onset and offset of the metronome, but were left uninstructed regarding an intra-SR-interval activity; they were told simply to listen silently to the stimuli and responses as they were presented. The Group-U condition, then, was the usual condition for aural pairedassociate learning except that the intra-SR interval was varied over the same intervals as in Groups $\mathbf{R}$ and $\mathbf{C}$.

In addition to the nine conditions of the foregoing factorial design ( 3 intra-SR intervals and 3 interval 
activities), a control group, Group $\mathbf{0}$, was included for which the intra-SR interval was of zero length. For this group, $E$ read the stimulus and response members in immediate succession at a $2.5-\mathrm{sec}$ rate. This rate was chosen because the resulting interval between the response of one pair and the stimulus of the next closely approximated the corresponding interval for the other nine conditions. By use of this last condition, the effectiveness of stimulus repetition (Group $R$ ) in maintaining availability can be assessed.

On test trials, all $S \mathrm{~s}$ were treated identically: the stimuli were spelled aloud by $E$ at a 2 -sec rate, thus leaving approximately $1.5 \mathrm{sec}$ for $S$ to respond with an adjective. The intertrial interval was $4 \mathrm{sec}$ for all conditions, during which time $S$ was alerted as to whether the upcoming trial was to be a study or a test trial. Upon completion of the 15th test trial, $S$ was asked, without forewarning, to recall as many of the stimulus members as he could in any order they happened to come to mind. A 2-min period was allowed for recall.

Eight different presentation orders were prepared such that for any given list every pair appeared at each position during the course of learning. The start order was varied from $S$ to $S$ within each condition. All experimental materials were read to $S$ from a Stowe memory drum by $E$ via a microphoneearphone link. The responses of $S$ on test trials were recorded on tape and transcribed later under more leisurely conditions.

Subjects. There were $16 \mathrm{Ss}$ in each of the 10 experimental conditions. The conditions were assigned randomly to $S \mathrm{~s}$ as they appeared in the laboratory with the restriction that a given condition was not assigned for the $n$th time until all other conditions had been assigned $n-1$ times. The 160 Ss were undergraduates at the University of Michigan who volunteered for paid participation.

\section{RESUlts}

The basic datum is the number of correct responses an $S$ made per level of PR. Since there were two pairs per PR level and 15 test trials, the maximum possible score per $S$ per PR level is 30 .

The effects of $P R$ on learning for Groups $R, C$, and $U$ are shown in Fig. 1. An overall analysis of variance affirms that both $P R$ and intra-SR activity were highly significant factors, $F(3,405)=46.64$, and $F(2,135)=$ 106.35, respectively, $p<0.001$ for both; but at the same time noninteracting factors,

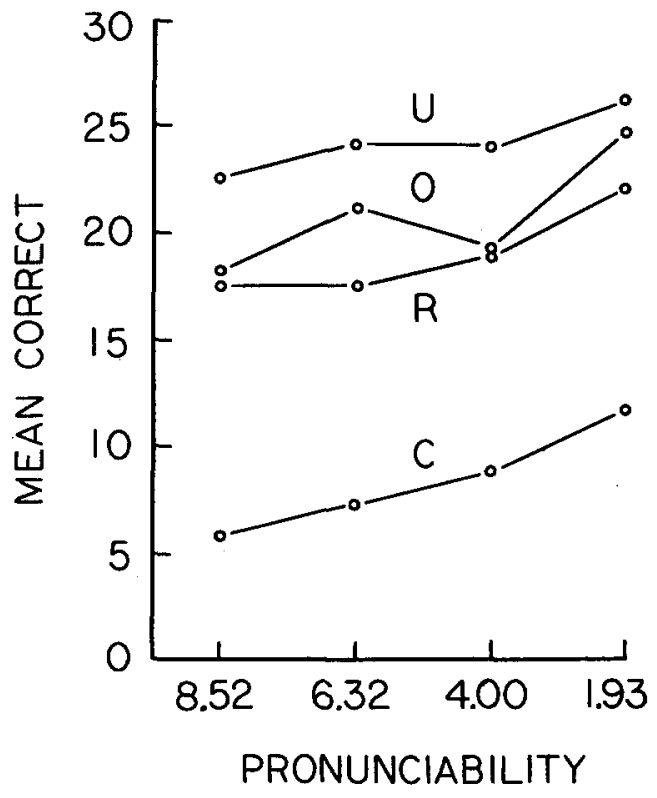

FIG. 1. Mean number correct responses per $S$ as a function of pronunciability (PR) with intra-SR activity as a parameter.

$F(6,405)=1.93, p>0.05$. Intra-SR interval, however, had no effect on learning and did not interact with either PR or intra-SR activity (all $F$ s less than 1 ). The data points for Groups $R, C$, and $U$ in Fig. 1 are what results from collapsing intervals.

In view of the interpretative importance of a possible interaction between Groups $R$ and C over levels of PR, further subanalyses were carried out. Considering only these two groups, an interaction between PR and intraSR activity again failed to appear, $F<1$. Even when this interaction was sought at the extreme levels of PR (8.52 and 1.93) under the longest intra-SR interval $(6 \mathrm{sec})$, statistical significance was not forthcoming, $F(1,30)=3.53, p>0.05$. Thus, as is visually evident in Fig. 1, PR did not have an effect on Group $C$ that was different from its effect on Group $R$.

Also plotted in Fig. 1 are the data for Group $O$, the zero intra-SR-interval condition. Since there were $16 \mathrm{Ss}$ in Group $\mathrm{O}$ but $48 \mathrm{Ss}$ in Groups $\mathrm{R}$ and $\mathrm{U}$ after collapsing intervals, Dunnett's (1955) test was used to 


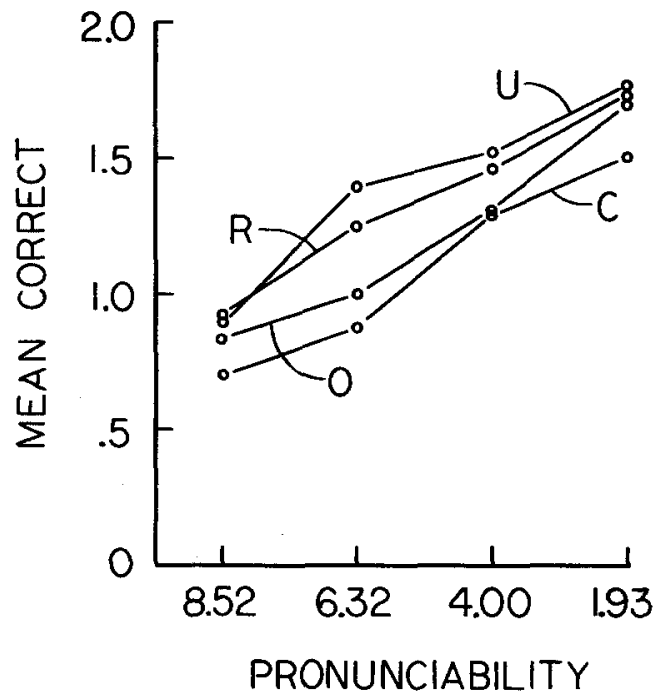

Frg. 2. Mean number correctly recalled as a function of pronunciability (PR) with intra-SR activity as a parameter.

compare Group O with Groups R and U. All differences between Group $O$ and Subgroups $2 \mathrm{R}, 4 \mathrm{R}$, and $6 \mathrm{R}$ are statistically nonsignificant $(p>0.05)$; similarly for the difference between Group $O$ and Subgroup 2U. Subgroups $4 \mathrm{U}$ and $6 \mathrm{U}$, however, are significantly superior to Group $O(p<0.05)$.

Regarding the un-forewarned free recall of the stimuli at the end of learning, the $2 \mathrm{~min}$ allowed proved to be more than adequate for all $S$ s. The results are shown in Fig. 2, where recall performance is plotted as a function of PR for Groups $U, R$, and C. Since there were two stimuli per PR level, the maximum possible score per $S$ per PR level is 2 . An overall analysis of variance indicates that both PR and intra-SR activity were significant, $F(3,405)=48.62, \quad p<0.001$, and $F(2,135)=6.10, \quad p<0.005, \quad$ respectively, but noninteracting $(F<1)$ factors in determining post-learning stimulus availability. As in the learning data, intra-SR interval played no discernible role $(p>0.20$ for all sources of variance associated with interval length).

Inclusion of the recall data of the Group-O condition in Fig. 2 shows that stimulus repetition (Group $R$ ) and uninstructed activity
(Group U) led to superior stimulus availability, while counting activity (Group C) produced inferior availability compared to Group O. However, although intra-SR activity is a large effect, the Group $O$ data, lying as they do central to the other groups, do not differ significantly from any of the other groups (using Dunnett's precedure).

\section{Discussion}

The two important findings of the present experiment seem to be, first, that stimulus repetition serves reasonably well to maintain stimulus availability, as evidenced by the closeness of the Group- $R$ data points to those of Group $O$ in Fig. 1, and, second, that the performance decrement due to counting (Group C) is not differential according to PR. If forgetting proceeds according to pronunciability and if this effect is to be manifested in paired-associate learning, then the difference between Groups $R$ and $C$ must be greater for pairs with difficult-to-pronounce stimuli than for those with easy-to-pronounce stimuli. This is because for Group $R$ the stimuli were maintained in availability over the intra-SR interval while for Group $\mathrm{C}$ conditions were optimal for differential forgetting. Thus the present experimental design makes the interaction of Groups $R$ and $C$ over levels of PR highly probable given the truth of the differential availability hypothesis. The failure to find this predicted effect means that stimulus availability, in the sense of recallability, either (a) has nothing to do with paired-associate learning or (b) is not a function of PR.

The latter alternative can be easily eliminated for several reasons. The free recall at the end of learning demonstrated that the stimuli were available in direct accordance with the ease of their pronunciation. This was a marked effect. Furthermore, there are independent data on the role of meaningfulness in the short-term memory for trigrams. As stated earlier, Lindley (1963), Murdock (1961), and Peterson et al. (1961) have 
shown that extent of loss is inversely related to meaningfulness. Thus rejection of the alternative that stimulus availability is not a function of $P R$ is justified.

The other alternative mentioned above, that stimulus availability has nothing to do with paired-associate learning, while in itself a negative proposition, therefore remains. Besides the present study, corroboration for this conclusion follows from an experiment by Schulz and Martin (1964). They showed that differential manipulation of trigram recallability over several levels of PR by means of 30 trials of aural familiarization did not transfer differentially to subsequent pairedassociate learning where the familiarized trigrams had become stimuli. Accumulating evidence is thus forcing denial of availability as the mechanism underlying stimulus-PR effects, and stimulus-meaningfulness effects in general, in paired-associate learning.

The inability to explain stimulus-meaningfulness effects in terms of stimulus availability notwithstanding, the problem of how, or why, this effect obtains remains a pressing one. The resolution that suggests itself to the present writer is the following. Imagine a $2 \times 3$ contingency table where the two rows represent the two major aspects intended by the meaningfulness of a verbal unit, the number of associations it elicits and its degree of integration in the sense of ordinary serial learning, and the three columns the three stages of information processing that might be separately affectable by various experimental variables, input (reception), storage (memory), and output (uttering). Assumption: meaningfulness in the sense of number of associations affects storage; meaningfulness in the sense of serial integration affects output.

On this view, it is stimulus meaningfulness in the sense of number of associations that affects the association-formation phase of paired-associate learning. This is because what response goes with what stimulus is a relationship that must be remembered in the either facilitating or interfering context of the associations elicited by the stimulus. On the other hand, overt recallability of the stimulus member of a pair is additionally determined by the serial-integration aspect of meaningfulness. Thus, recallability as determined by meaningfulness is seen as mediated by both the number-of-associations and the serial-integration aspects of meaningfulness, while the effect of stimulus meaningfulness on paired-associate learning is seen as mediated by the number-of-associations aspect only.

\section{REFERENCES}

Dunnetr, C. W. A multiple comparison procedure for comparing several treatments with a control. J. Amer. statist. Ass., 1955, 50, 1096-1121.

HAaGeN, C. H. Scaling the principal attributes of words to be used in experiments in verbal learning. Masters thesis, State University of Iowa, 1942.

LindLEx, R. H. Effects of controlled coding cues in short term memory. J. exp. Psychol., 1963, 66, 580-587.

Martin, E., ANd Schulz, R. W. Aural pairedassociate learning: pronunciability and the interval between stimulus and response. $J$. verb. Learn. verb. Behav., 1963, 1, 389-391.

Murdock, B. B., JR. The retention of individual items. J. exp. Psychol., 1961, 62, 618-625.

Noвle, C. E. Meaningfulness and familiarity. In C. N. Cofer and B. S. Musgrave (Eds.), Verbal behavior and learning: problems and processes. New York: McGraw-Hill, 1963. Pp. 76-119.

Peterson, L. R., Peterson, M. J., and Miller, G. A. Short-term retention and meaningfulness. Canad. J. Psychol., 1961, 15, 143-147.

Schulz, R. W., AND MARTin, E. Aural pairedassociate learning: stimulus familiarization, response familiarization, and pronunciability. $J$. verb. Learn. verb. Behav., 1964, 3, 139-145.

ThoRndike, E. L., AND LORGE, I. The teacher's word book of 30,000 words. New York: Columbia University Press, 1944.

UNderwood, B. J., AND Schulz, R. W. Meaningfulness and verbal learning. Philadelphia: Lippincott, 1960.

(Received September 8, 1964) 